\title{
A hybrid brain-computer interface based on the fusion of electroencephalographic and electromyographic activities
}

\author{
Robert Leeb, Hesam Sagha, Ricardo Chavarriaga and José del R Millán \\ Chair in Non-Invasive Brain-Machine Interface, Center for Neuroprosthetics, Institute of Bioengineering, \\ School of Engineering, Ecole Polytechnique Fédérale de Lausanne, CH-1015 Lausanne, Switzerland \\ E-mail: robert.leeb@epfl.ch
}

Received 30 July 2010

Accepted for publication 23 November 2010

Published 24 March 2011

Online at stacks.iop.org/JNE/8/025011

\begin{abstract}
Hybrid brain-computer interfaces (BCIs) are representing a recent approach to develop practical BCIs. In such a system disabled users are able to use all their remaining functionalities as control possibilities in parallel with the BCI. Sometimes these people have residual activity of their muscles. Therefore, in the presented hybrid BCI framework we want to explore the parallel usage of electroencephalographic (EEG) and electromyographic (EMG) activity, whereby the control abilities of both channels are fused. Results showed that the participants could achieve a good control of their hybrid BCI independently of their level of muscular fatigue. Thereby the multimodal fusion approach of muscular and brain activity yielded better and more stable performance compared to the single conditions. Even in the case of an increasing muscular fatigue a good control (moderate and graceful degradation of the performance compared to the non-fatigued case) and a smooth handover could be achieved. Therefore, such systems allow the users a very reliable hybrid BCI control although they are getting more and more exhausted or fatigued during the day.
\end{abstract}

(Some figures in this article are in colour only in the electronic version)

\section{Introduction}

Brain-computer interfaces (BCIs) allow disabled people to establish a new communication channel between the human brain and a machine [1]. This communication is based on the analysis of electrophysiological brain signals recorded by the electroencephalogram (EEG). Although BCI technology has shown impressive progress in the last few years [2], it cannot be compared to non-BCI control channels in terms of performance and interaction speed. Therefore, the development of practical BCIs for disabled people should allow them to use all their remaining functionalities as control possibilities and to use the currently best available ones. Especially since the physical and mental conditions of a patient (e.g. early stage of amyotrophic lateral sclerosis) are changing over the day, various control strategies could be applied, e.g. sometimes muscular activity would be available (most likely in the morning when they are not exhausted), whereas at other times maybe only brain signals can be voluntarily controlled.

Such a combination and parallel usage of at least one BCI and at least one additional communication (e.g. another physiological signal or special assistive input devices such as joysticks, switches) is called a hybrid BCI [3, 4]. Generally these control channels can operate different parts of the assistive device or all of them could be combined to allow users to smoothly switch from one control channel to the other, depending on their preference and performance. We can assume that such a hybrid BCI will improve the quality of life of a patient. The following examples of hybrid BCIs can already be found in the literature: based on multiple brain signals, such as the combination of a motor imagery (MI)-based BCI with a steady-state visual evoked potential (SSVEP)-based BCI [5], or the combination of an MI BCI with error potential (ErrP) detection and correction of false 


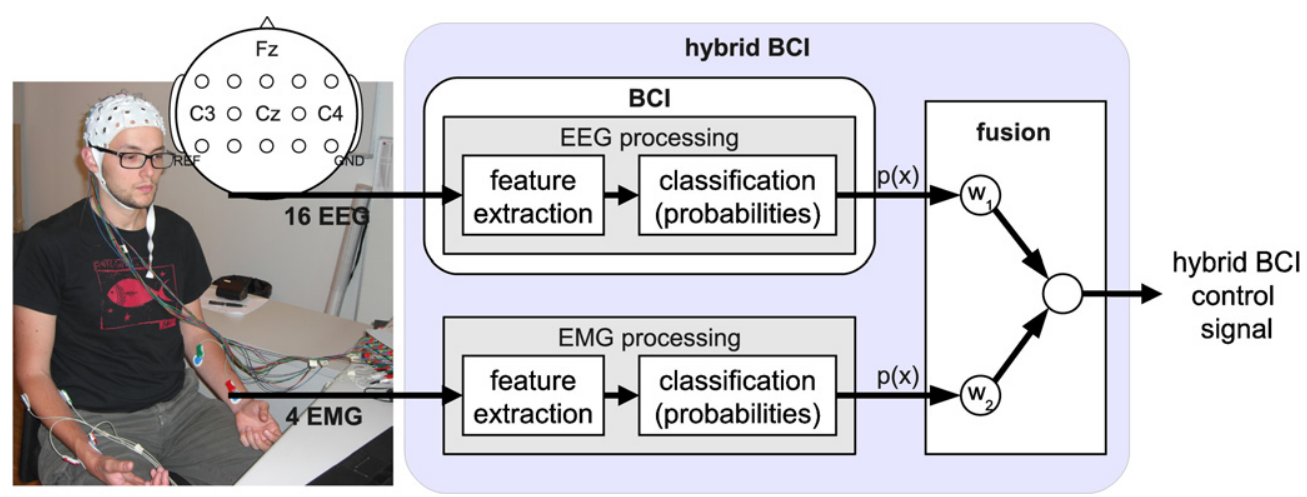

Figure 1. The photograph shows a subject wearing the cap with 16 EEG electrodes over the motor cortex and 4 EMG channels at the flexor and extensor of the left and right forearm. The diagram explains the processing and fusion principle of muscular and brain activities in a hybrid BCI.

mental commands [6], or the combination of a SSVEP BCI with a heart rate controlled on/off switch [7].

In the presented hybrid BCI framework we want to explore the parallel usage of EEG and electromyographic (EMG) activity. The control abilities of both channels will be fused, so that subjects could achieve a good control of their hybrid BCI independently of their level of muscular fatigue.

\section{Methods}

\subsection{Experimental paradigm and subjects}

Every trial in this synchronous BCI recording started with a fixation cross over $3 \mathrm{~s}$ on a screen in front of the participants. Afterwards the cue - an arrow pointing to the left or rightwas displayed for $5 \mathrm{~s}$ and the subjects had to perform repetitive movements with their left or right hand depending on this cue, i.e. clutching the hand in a fist. The trial ended with a random pause between 1.5 and $2.5 \mathrm{~s}$. In total, four runs ( 5 min each) with 15 trials for right and 15 trials for left motor execution were recorded per subject, resulting in 60 trials per class. The EEG and EMG data were recorded with two g.USBamps (gtec medical engineering, Schiedelberg, Austria) using a sampling rate of $512 \mathrm{~Hz}$ and a band pass filter between 0.1 and $100 \mathrm{~Hz}$ with activated notch filter.

Twelve healthy subjects (mean age $28.9 \pm 5.3$ years) participated in the recordings. Unfortunately, one subject had very strong electrode movement artefacts in the EEG and had to be removed from further analysis. Furthermore, another subject was excluded because no EEG classifier with a performance better than the chance level could be identified and a third one because an EMG electrode lost contact during the experiment and no signal could be recorded.

\subsection{Processing steps}

The EEG was acquired monopolarly over the motor cortex with 16 electrodes (see figure 1). From the Laplacian filtered EEG, the power spectral density (PSD) was estimated in the band 4-48 Hz with $2 \mathrm{~Hz}$ resolution over the last second [8]. The
PSD features were then estimated every $62.5 \mathrm{~ms}$ (i.e. 16 times per second) using the Welch method with 5 overlapped (25\%) Hanning windows of $500 \mathrm{~ms}$. Canonical variate analysis was used to select subject-specific features that maximized the separability between the different tasks and stable features (according to cross validation on the training data) were used to train a Gaussian classifier [9]. Decisions with low confidence on the probability distribution are filtered out if they were not above a given rejection threshold. Finally, temporary evidence about the executed task was accumulated using an exponential smoothing probability integration framework [10].

The EMG was acquired bipolarly over the flexor and extensor of the left and right forearm (see figure 1). The prehensile EMG activities were rectified and averaged over $0.3 \mathrm{~s}$ to extract the envelopes. The resulting features were subject-specific thresholded per channel, normalized and classified based on maximum distance. For estimating the EMG thresholds a short recording with two left- and two right-hand movements was performed before the experiment. The EMG features were calculated and only the background activity during the non-execution time was extracted. The thresholds were finally set to the mean average plus three times the standard deviation (SD) of these periods, in order that most of the spontaneous EMG activity during the nonexecution time is not detected (similar to [11]).

\subsection{Fusion techniques}

The fusion module on the right side of figure 1 deals with the probabilities of all the preceding classification modules, in our case the EEG and EMG classifiers. In this implementation we apply an approach with static fusion rules, since we assume that both inputs can be used all the time (the classifier output rates are $16 \mathrm{~Hz}$ ).

Two fusion techniques have been tested: the first approach uses equally balanced weights between the two classifiers and the second one applies the naïve Bayesian fusion approach [12]. The Bayesian fusion considers the conditional model $P\left(C \mid O_{1}, O_{2}\right)$ to combine the decisions from two sources (EEG and EMG), where $\mathrm{C}$ denotes the class (left or right), and $O_{1}$ and $O_{2}$ are the decisions from the 
two classifiers corresponding to EEG and EMG, respectively. According to the Bayes rule, we have

$$
P\left(C \mid O_{1}, O_{2}\right) \propto P(C) P\left(O_{1}, O_{2} \mid C\right) .
$$

Assuming that the two sources are independent, we can write

$$
P\left(O_{1}, O_{2} \mid C\right)=P\left(O_{1} \mid C\right) P\left(O_{2} \mid C\right) .
$$

Finally we can state

$$
C_{\text {out }}=\operatorname{argmax}_{c}\left(P(C=c) P\left(O_{1} \mid C=c\right) P\left(O_{2} \mid C=c\right)\right)
$$

with $c \in\{$ left, right $\}$. We assume that the prior of the two classes $P(C=c)$ are the same. Finally $P\left(O_{1} \mid C=c\right)$ and $P\left(O_{2} \mid C=c\right)$ are the probability values from the confusion matrix estimated using the training data.

\subsection{Fatigue simulation}

In this experiment the influence of muscular fatigue on the fused EEG and EMG performance was investigated. To this end, we simulated fatigue-induced EMG changes by degrading the amplitudes of the EMG channel over the experimental time (attenuation from $10 \%$ up to $100 \%$ ), and thereby making the EEG activity more and more important for the fusion. Nevertheless the same classifier weights for EEG and EMG and the same fusion rules were applied. This simulates the realistic situation of a patient who becomes more and more fatigued over the day.

In the literature, both decreases and increases in EMG amplitude have been found as a sign of fatigue [13, 14]. A detected increase is mostly triggered by the recruitment of additional motor units to compensate the decrease of force. As fatigue increases, EMG amplitude also increases initially (if the participant is trying to produce the same force) and then it strongly decreases [13]. A better measure for fatigue would be the intracellular action potential change, which cannot be recorded with surface EMG electrodes. A further possibility of detecting fatigue is the analysis of the median or mean frequency of the myoelectric signal power spectrum, which is predominantly linearly decreasing during fatigue [15].

Before performing our experiment we investigated the validity of using amplitude reduction as fatigue simulation. Thereby the subjects were repeatedly executing left and right hand movements with hand grippers, until they could not do the exercise any longer. The recorded EMG showed first a stable amplitude; with advancing fatigue the amplitude level first increased by a factor of 2 and then decreased to $10 \%$ (similar to the results reported in [13]). We also found the decrease in the median frequency at later fatigue stages.

\section{Results}

In our experiment, the BCI can be controlled either by a single modality (EEG or EMG) or by the fused activity of both. In total we have compared six different conditions in figure 2: two single modalities and four fused activities with increasing levels of muscular fatigue (i.e. $0 \%, 10 \%, 50 \%, 90 \%$ attenuation

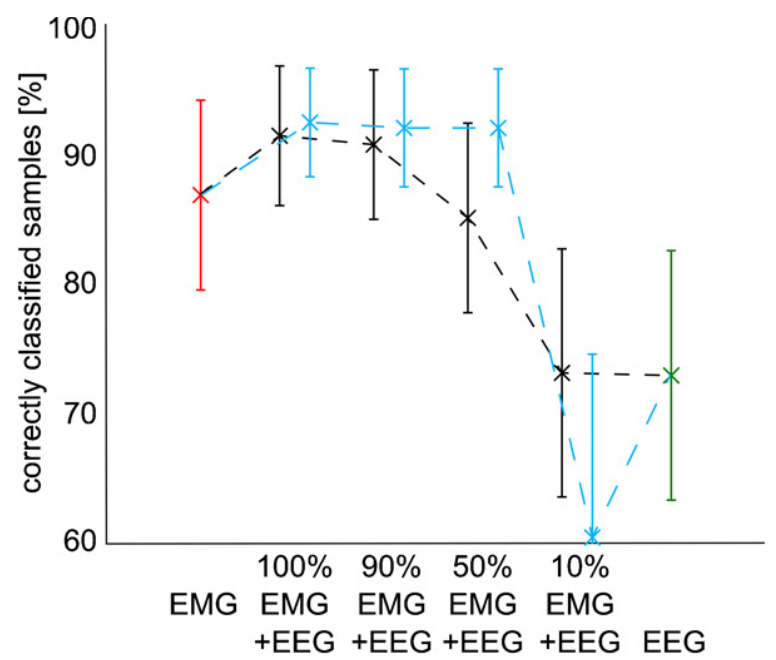

Figure 2. Mean $\pm \mathrm{SD}$ of correctly classified samples over the whole task period ( $0-5 \mathrm{~s})$ for the six conditions. The leftmost (red) and rightmost (green) conditions correspond to the single modalities, EMG and EEG, respectively. The four conditions in the middle correspond to the fusion of EEG and EMG with different levels of remaining amplitude (i.e. 0\%, 10\%, 50\%, 90\% attenuation). For each of these conditions we provide two performances according to the fusion modality: simple fusion (left side in black) and Bayesian fusion (right side in blue).

of EMG amplitude). The reported performance measures were calculated based on the number of correctly classified samples over the task time $(0-5 \mathrm{~s})$ and were evaluated by a $4 \times 4$ cross validation.

The average performance of all subjects for the EEG activity alone was $73 \%$ and for EMG activity alone was $87 \%$. In the first fusion approach (equally weighted sources) the fused activity achieved an increase to $91 \%$. Remarkably, thanks to the fusion of EEG and EMG, increasing muscular fatigue (from $10 \%$ to $50 \%$ to $90 \%$ attenuation) led to a moderate and graceful degradation of performance: $90 \%, 85 \%$ and $73 \%$ accuracy, respectively. It is worth noting that in the case of fusion with only $10 \%$ of EMG amplitude $(90 \%$ attenuation), the performance is the same as for EEG alone despite the fact that the fusion weights are the same over all conditions.

The second fusion technique based on the Bayesian approach achieved similar results but with smaller SD (see figure 2). Interestingly the Bayesian fusion performance is very stable over the first three fatigue conditions. Especially in the 50\% EMG condition, a tremendous increase could be achieved compared to the other fusion technique (statistically significant from $85.1 \%$ to $92.0 \%$ ). In contrast, in the last condition (90\% EMG attenuation) the Bayesian approach failed and had a result of $60.4 \%$, which is worse than EEG alone. The reason is that the confusion matrices of the Bayesian fusion have been calculated using a non-fatigued subject and the method assumes that the sources do not change over time. However, a strong level of EMG fatigue leads to almost a removal of this source, thus causing the significant performance decrease. 

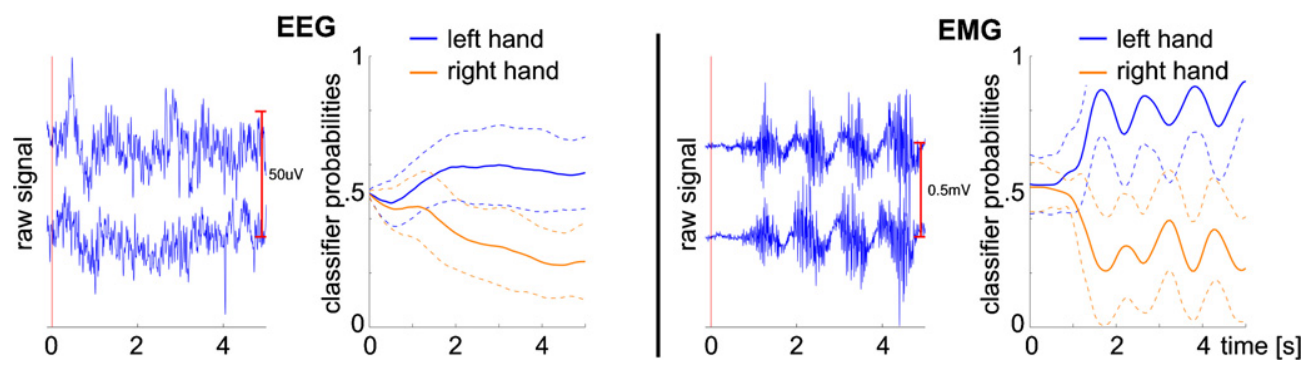

Figure 3. Examples of raw signals and averaged classifier outputs (integrated probabilities; mean in solid lines \pm SD in dotted lines) of EEG (left) and EMG (right). The cue appeared at $0 \mathrm{~s}$.

\section{Discussion}

This experiment demonstrates the benefits of a hybrid BCI. Multimodal fusion of muscular and brain activity yielded better and more stable performance compared to the single conditions. Furthermore, the increasing muscular fatigue led only to a moderate and graceful degradation of performance compared to the non-fatigued case. Therefore, such a system allows the user a very reliable hybrid BCI control, even though she/he is getting more and more exhausted or fatigued during the day.

Comparing the behaviour of the two fusion techniques, it is obvious that the Bayesian fusion achieved a constant performance over a wide range of muscular fatigue, compared to the steadily decreasing performance in the case of the simple fusion. However, the Bayesian approach yielded the worst performance in case of $10 \% \mathrm{EMG}$, even lower than the EEG alone condition. This behaviour can be explained by the dominance dependence of the Bayesian fusion approach on the EMG classifier output. For fatigue levels of 50\% and lower the output of the classifier was still reliable and therefore the Bayesian approach achieved better results. However, in the conditions in which the quality of the EMG input signals dropped below a certain threshold the results were worse. The reason is the strong violation of the assumption that the input patterns are stationary over time, necessary to compute the Bayesian confusion matrices. This problem could be overcome by adapting the way we consider the contribution of the different modalities. In this work we have chosen a static approach (computed once and kept constant over time). Instead we should dynamically update these coefficients based on the reliability of the input channels, or the confidence/certainty the system has on its outputs.

Surprisingly, the fused activity resulted in a $6 \%$ improvement in classification compared with the EMG alone condition. One may expect that EMG classification leads to a perfect classification of $100 \%$. The reason for the 'nonperfect' classification of the single EMG condition is based on the fact that the movements were repetitively executed and that the number of correctly classified samples over the whole task time is used as a performance measure. A glance at the raw signals and the extracted classifier outputs exemplifies the behaviour (see figure 3). The EEG classifier had a smooth but stable improvement over the trial time compared to the fast and strong but fluctuating response of the EMG classifier, which also had a large variation over time. The EMG fluctuation over time can be explained by the repetitive execution of the hand movements during the task time. Sometimes the subject executes the movements and sometimes pauses them. Thereby the EMG power drops below the detection threshold and therefore is counted as not detected. On the other side, repetitive movements are commonly used in BCI research, since they lead to more discriminative and stable EEG patterns.

Generally speaking, besides muscular fatigue a mental fatigue could also appear. This would influence the reliability of the EEG signal in a similar way as the simulated muscular fatigue influenced the performances of the EMG channel. Such a reliability could be estimated from supervision signals such as cognitive mental states in the case of EEG (e.g. fatigue, error potentials) and physiological parameters (e.g. median frequency of the myoelectric signal power spectrum in the case of muscular fatigue). Another possibility is to analyse the performance of the individual classifiers in achieving the task (e.g. stability over time, influence of noise, etc) and thereby adapt the fusion weights.

In our future work we will implement hierarchical probabilistic approaches, where each channel is modelled independently and exploits appropriate priors. Thereby the possibility of monitoring mental states and other physiological parameters-which are a source of meta-control signals for weighting the contributions of the single modalities and/or switching between them-can be incorporated. Online monitoring of mental states is indeed a critical source of information to facilitate and improve BCI use over long periods of time. EEG correlates of mental states associated with fatigue, attention and workload can modify online the behaviour of the BCI in two ways. The first one is rather direct via shared control [3] by setting the level of assistance received by the user to compensate for his/her reduced attention due to an increase in fatigue or workload. Similarly, the level of assistance would decrease whenever the user could sustain a high level of attention. The second way we can incorporate measures of mental states is through the hybrid architecture where these measures weight the contribution of the EEG channel. Indeed, these measures are a good estimator of the reliability of the EEG channel: for instance, if the user has a high attentional level to cope with the workload of the task, then we can trust the output of the EEG channel; otherwise, we might not trust it to such an extent [3].

Finally, patients with progressive loss of muscular activity (as in muscular dystrophy, amyotrophic lateral sclerosis and 
spinal muscular atrophies) could benefit from such a hybrid BCI with dynamic fusion. For example, during early hybrid BCI training the user could still exploit her/his residual motor functions, while with increasing long-term use of the assistive product the transition between the hybrid assistive device and pure BCI (when muscular activity is too weak to operate them) would be smooth. Furthermore, with the proposed hybrid approach of smoothly merging the various inputs, we can expect that users can naturally cope with fatigue (muscular as well as mental) as they can rely on the best input at any time which would allow them to recover quickly.

\section{Acknowledgments}

This work is supported by the European ICT programme projects TOBI: Tools for Brain-Computer Interaction (FP7-224631) and OPPORTUNITY: Activity and Context Recognition with Opportunistic Sensor Configurations (FP7225938). This paper only reflects the authors' views and funding agencies are not liable for any use that may be made of the information contained herein. The authors are grateful to Tom Carlson for proofreading the manuscript and to all CNBI collegues for their help in setting up the BCI.

\section{References}

[1] Wolpaw J R, Birbaumer N, McFarland D J, Pfurtscheller G and Vaughan T M 2002 Brain-computer interfaces for communication and control Clin. Neurophysiol. $113767-91$

[2] Allison B Z, Wolpaw E W and Wolpaw J R 2007 Brain-computer interface systems: progress and prospects Expert Rev. Med. Devices 4 463-74

[3] Millán JdR, Rupp R, Müller-Putz G, Murray-Smith R, Giugliemma C, Tangermann M, Vidaurre C, Cincotti F, Kübler A, Leeb R, Neuper C, Müller K and Mattia D 2010 Combining brain-computer interfaces and assistive technologies: state-of-the-art and challenges Front. Neuroprost. 4161
[4] Pfurtscheller G, Allison B, Bauernfeind G, Brunner C, Solis Escalante T, Scherer R, Zander T, Müller-Putz G, Neuper C and Birbaumer N 2010 The hybrid BCI Front. Neurosci. 442

[5] Brunner C, Allison B Z, Krusienski D, Kaiser V, Müller-Putz G, Pfurtscheller G and Neuper C 2010 Improved signal processing approaches in an offline simulation of a hybrid brain-computer interface J. Neurosci. Methods 188 165-73

[6] Ferrez P W and Millán J 2008 Error-related EEG potentials generated during simulated brain-computer interaction IEEE Trans. Biomed. Eng. 55 923-9

[7] Scherer R, Müller-Putz G R and Pfurtscheller G 2007 Self-initiation of EEG-based brain-computer communication using the heart rate response J. Neural Eng. 4 L23-9

[8] Galán F, Nuttin M, Lew E, Ferrez P W, Vanacker G, Philips J and Millán J 2008 A brain-actuated wheelchair: asynchronous and non-invasive brain-computer interfaces for continuous control of robots Clin. Neurophysiol. $1192159-69$

[9] Millán J, Ferrez P W, Galán F, Lew W and Chavarriaga R 2008 Non-invasive brain-machine interaction Int. J. Pattern Recognit. Artif. Intell. 22 959-72

[10] Perdikis S, Tavella M, Leeb R and Millán J 2010 Feedback controller for mental imagery BCI Proc. TOBI Workshop 2010: Integrating Brain-Computer Interfaces with Conventional Assistive Technology $\mathrm{p} 71$

[11] Leeb R, Friedman D, Müller-Putz G R, Scherer R, Slater M and Pfurtscheller G 2007 Self-paced (asynchronous) BCI control of a wheelchair in virtual environments: a case study with tetraplegics Comput. Intell. Neurosci. 79642 1-8

[12] Rogova G and Nimier V 2004 Reliability in information fusion: literature survey Proc. Int. Conf. Inf. Fusion pp 1158-65

[13] Dimitrova N A and Dimitrov G V 2003 Interpretation of EMG changes with fatigue: facts, pitfalls and fallacies J. Electromyogr. Kinesiol. 13 13-36

[14] Gerdle B, Larsson B and Karlsson S 2000 Criterion validation of surface EMG variables as fatigue indicators using peak torque: a study of repetitive maximum isokinetic knee extensions J. Electromyogr. Kinesiol. 10 225-32

[15] Viitasalo J and Komi P 1977 Signal characteristics of EMG during fatigue Eur. J. Appl. Physiol. 37 111-21 